\title{
Acute inhibition of TAK1 protects against neuronal death in cerebral ischemia
}

\author{
M Neubert ${ }^{1,3}$, DA Ridder ${ }^{1,3}$, P Bargiotas ${ }^{1}$, S Akira ${ }^{2}$ and M Schwaninger ${ }^{*, 1}$
}

Neuronal apoptosis contributes to ischemic brain damage and neurodegenerative disorders. Key regulators of neuronal apoptosis are the transcription factor NF- $\kappa$ B and the MAP kinases p38/MAPK and JNK, which share a common upstream activator, the mitogen-activated protein kinase kinase kinase (MAP3K) TGF $\beta$-activated kinase 1 (TAK1). Here we investigate the function of TAK1 in ischemia-induced neuronal apoptosis. In primary cortical neurons, TAK1 was activated by oxygen glucose deprivation (OGD), an in vitro model of cerebral ischemia. We found that short-term inhibition of TAK1 protected against OGD in vitro and reduced the infarct volume after middle cerebral artery occlusion in vivo. Prolonged inhibition or deletion of the TAK1 gene in neurons was, however, not protective. Short-term, but not prolonged inhibition of TAK1 interfered with the activation of p38/MAPK and JNK by OGD, the induction of the pro-oxidative genes Cox-2, Nox-2, and p40 phox and the formation of superoxide. We found that prolonged TAK1 inhibition upregulated another MAP3K, apoptosis signal-regulating kinase-1, which is able to compensate for TAK1 inhibition. Our study demonstrates that TAK1 is a central target for short-term inhibition of key signaling pathways and neuroprotection in cerebral ischemia.

Cell Death and Differentiation (2011) 18, 1521-1530; doi:10.1038/cdd.2011.29; published online 8 April 2011

Stroke is the most common neurological disease and the second leading cause of death in western countries. ${ }^{1}$ Presently, treatment options are still not satisfactory. ${ }^{2}$ To improve this situation, the molecular events that lead to tissue demise and neuron loss in cerebral ischemia need to be elucidated. Neurons die of apoptosis in the border zone of an ischemic stroke, the so-called penumbra. In the context of neuronal apoptosis, NF- $\kappa$ B and the MAP kinases JNK and p38/MAPK are major regulators of gene transcription.

$\mathrm{NF}-\kappa \mathrm{B}$ is activated in neurodegenerative disorders and in ischemic stroke. ${ }^{3,4}$ Although the mechanism of NF- $\kappa$ B activation in cerebral ischemia is still not completely understood, there is evidence that the upstream kinase IKK is involved. ${ }^{5-7} \mathrm{NF}-\kappa \mathrm{B}$ is well known for its anti-apoptotic action. Surprisingly, however, $\mathrm{NF}-\kappa \mathrm{B}$ promotes neuronal apoptosis in cerebral ischemia and neurodegenerative disorders. In line with this concept, deletion of the NF- $k$ B subunits $p 50$ or p65 reduced the infarct size in a mouse model of stroke. ${ }^{3,8,9}$ Furthermore, inhibition or deletion of the upstream kinase IKK protected against acute neurodegeneration in cerebral ischemia. ${ }^{6}$ In Parkinson's and Huntington's disease, a detrimental effect of IKK has been reported. ${ }^{4,7}$

In parallel to the NF- $\kappa \mathrm{B}$ pathway, cerebral ischemia activates the MAP kinases JNK and p38/MAPK ${ }^{10}$ that signal to the transcription factor AP-1 and other targets. Indeed, ample evidence documents that JNK aggravates ischemic brain damage and neurodegenerative disorders. ${ }^{11,12}$ Selective inhibition of p38/MAPK was also shown to reduce the infarct size. ${ }^{13}$
In view of the important role of NF- $\kappa$ B, JNK, and p38/MAPK signaling in neuronal apoptosis, it is interesting to note that the $\mathrm{NF}-\kappa \mathrm{B}$ and MAP kinase signaling pathways are connected at several levels. Target genes of NF- $\kappa$ B inhibit JNK signaling. ${ }^{14,15}$ Therefore, inhibiting IKK promotes overactivation of AP-1 in cerebral ischemia, ${ }^{16}$ which may neutralize the efficacy of IKK inhibition. However, there is also a mechanism that activates all three pathways at the same time; it involves the common upstream kinase, TGF $\beta$-activated kinase 1 (TAK1). TAK1 is a mitogen-activated protein kinase kinase kinase (MAP3K). In response to IL- $1 \beta$, TNF, TGF $\beta$, and other stimuli, TAK1 activates the MAP2Ks MKK4/7 and MKK3/6, which activate JNK and p38/MAPK, respectively. In addition, TAK1 is responsible for activating IKK and the canonical NF- $k B$ signaling pathway. TAK1-deficient mice are embryonically lethal. ${ }^{17,18}$ Therefore, a conditional approach has been used to investigate the function of TAK1 in postnatal mice. This strategy showed that TAK1 is a key regulator of innate and adaptive immune responses. ${ }^{18}$ Furthermore, TAK 1 is essential for the survival of hematopoietic cells, hepatocytes, and keratinocytes. ${ }^{19,20}$ Deletion of TAK1 in liver parenchymal cells caused apoptosis and early onset hepatocarcinogenesis. ${ }^{1,21}$

Although TAK 1 is highly expressed in the brain, ${ }^{22}$ little is known about TAK 1 function in the central nervous system. It is also unclear whether TAK1 can be activated by ischemia. In this study, therefore, we aimed to characterize the role of TAK1 in neurons during cerebral ischemia. For this purpose, we inhibited TAK1 by a small-molecule compound and

\footnotetext{
${ }^{1}$ Department of Pharmacology, University of Heidelberg, Heidelberg, Germany and ${ }^{2}$ Institute for Microbial Diseases, Osaka University, Osaka, Japan

${ }^{*}$ Corresponding author: M Schwaninger, Institute of Experimental and Clinical Pharmacology and Toxicology, University of Lübeck, Ratzeburger Allee 160, Lübeck 23538, Germany. Tel: + 49451 5002681; Fax: + 49451 5003327; Email: markus.schwaninger@pharma.uni-luebeck.de

${ }^{3}$ These authors contributed equally to this work.

Keywords: TAK1; ischemia; neuroprotection

Abbreviations: ASK-1, apoptosis signal-regulating kinase 1; DHE, dihydroethidine; GSH, glutathione; OGD, oxygen glucose deprivation; MAP3K, mitogen-activated protein kinase kinase kinase; MCAO, middle cerebral artery occlusion; OZ, 5Z-7-oxozeaenol; TAK1, TGF $\beta$-activated kinase 1; TUNEL, terminal deoxynucleotidyl transferase-mediated dUTP-biotin nick end labeling

Received 10.8.10; revised 01.2.11; accepted 23.2.11; Edited by L Greene; published online 08.4.11
} 
deleted the kinase by a genetic approach. Our data demonstrate that acute inhibition of TAK1 provides neuroprotection in ischemia, whereas chronic inhibition is compensated by upregulation of the MAP3K apoptosis signal-regulating kinase-1 (ASK-1) and subsequent activation of JNK and p38/MAPK.

\section{Results}

Acute inhibition of TAK1 provides neuroprotection in cerebral ischemia. To discover whether cerebral ischemia activates TAK1 in primary cortical neurons, we used a pulldown kinase assay of TAK1 with MKK6 as substrate. Neurons treated with TNF $(10 \mathrm{ng} / \mathrm{ml})$ showed a slightly increased TAK1 activity that was blocked by a 1-h preincubation with the specific TAK1 inhibitor 5Z-7oxozeaenol (OZ, $600 \mathrm{nM}^{23}$ Figure 1a). After oxygen glucose deprivation (OGD), an in vitro model of cerebral ischemia, TAK1 was activated (Figure 1a). To investigate whether TAK1 influences neuronal apoptosis, we inhibited the kinase activity of TAK1 with OZ starting $1 \mathrm{~h}$ before OGD, and investigated apoptosis after a recovery period of $24 \mathrm{~h}$ by terminal deoxynucleotidyl transferase-mediated dUTP-biotin nick end labeling (TUNEL) staining. Indeed, we found fewer TUNEL-positive neurons after TAK1 was inhibited than in vehicle- (DMSO) treated cultures (Figure 1b). As TUNELpositive cells also showed a condensed nucleus in $4^{\prime}, 6$ diamidin-2-phenylindol (DAPI) staining (Figure 1b), we used nuclear staining by DAPI to detect apoptosis in subsequent experiments. By quantifying condensed apoptotic nuclei in cultures pretreated with increasing concentrations of $\mathrm{OZ}$, neuroprotection was confirmed, with the maximal effect at a concentration of $600 \mathrm{nM}$ (Figure 1c). To verify that the TAK1 inhibitor reduced apoptosis, we measured oligonucleosome accumulation in the cytoplasm as a sign of DNA fragmentation. Indeed, oligonucleosomes increased after OGD in control cultures, but not in cultures pretreated with $\mathrm{OZ}$ (Figure 1d). Taken together, these results indicate that inhibition of TAK1 shortly before OGD protects neurons from apoptosis.

To further investigate whether inhibition of TAK1 is also neuroprotective in an in vivo model of cerebral ischemia, we subjected mice to middle cerebral artery occlusion (MCAO). We found that intracerebroventricular injection of OZ (4 ng or $14 \mathrm{ng}$ ) at $20 \mathrm{~min}$ before MCAO significantly reduced the infarct volume (Figure 1e). This protective effect was dose dependent. To test whether the reduction in infarct size also has an impact on mouse behavior, we performed corner tests. ${ }^{24}$ Mice that were injected with the solvent DMSO showed an increased rate of right turnings after MCAO. This effect was lost after pretreatment with OZ (4 ng), suggesting that TAK1 inhibition improves the sensorimotor outcome (Figure 1f). In keeping with our findings, these results also demonstrate that activation of TAK1 contributes to ischemic brain damage.

Chronic inhibition of TAK1 is not neuroprotective. Acute TAK1 inhibition protected neurons in cerebral ischemia; therefore, we investigated whether genetic deletion of TAK1 had the same effect. As $\mathrm{TAK}^{-1-}$ mice die at
E9.5-E10.5, ${ }^{17,18}$ we used a conditional approach to delete TAK1 in neurons. For this, we infected neurons from TAK1 $1^{\mathrm{fl} / \mathrm{fl}}$ mice $^{18}$ with adenoviruses that express either only GFP (Ad-GFP) or the Cre recombinase together with GFP (Ad-Cre-GFP). At 4 days after infection, most neurons expressed GFP. At 10 days after infection, we performed immunoblotting of cell lysates to determine whether TAK1 was present. After infection with Ad-Cre-GFP, but not with the control virus Ad-GFP, no TAK1 protein was detected (Figure 2a). We used these cultures at 10 days after infection to further investigate whether deletion of TAK1 is neuroprotective in OGD. Unexpectedly, deleting TAK1 did not have any effect on the rate of neuronal apoptosis after OGD (Figure 2b). To exclude unspecific effects of OZ, we pretreated primary cortical TAK $1^{\mathrm{fl} / \mathrm{fl}}$ or $\mathrm{TAK} 1^{-/-}$neurons with either $\mathrm{OZ}$ or the solvent DMSO. In line with previous results, TAK $1^{\mathrm{fl} / \mathrm{fl}}$ neurons were protected by $\mathrm{OZ}$ in OGD. However, $\mathrm{OZ}$ did not have any effect on survival of TAK1 $1^{-1-}$ neurons, demonstrating that the protective effect of $\mathrm{OZ}$ depends on the presence of TAK1 (Figure 2c). To mimic the protracted time course of the genetic TAK1 deletion, we extended the $\mathrm{OZ}$ treatment to 10 days. OZ lost its protective action if treatment was prolonged to 10 days (Figure $2 \mathrm{~d}$ ). Thus, only acute but not chronic inhibition of TAK1 protects primary cortical neurons from OGD-induced apoptosis.

To test whether TAK1 deletion in vivo is beneficial during MCAO, we crossed TAK $1^{\text {fl/fl }}$ mice with mice carrying the allele for the inducible CreER ${ }^{\mathrm{T} 2}$ recombinase under control of the neuron-specific CaMKIl $\alpha$ promoter $\left(\mathrm{TAK} 1^{\mathrm{nKO}}\right) \cdot{ }^{25}$ Because tamoxifen affects ischemic brain damage, ${ }^{26}$ we postponed MCAO to 6 weeks after tamoxifen treatment. With this regimen, tamoxifen had no effect on the infarct size (data not shown). Immunoblotting showed significantly less TAK1 in the cortex of CaMKIl $\alpha$-CreER ${ }^{\mathrm{T} 2}$-positive mice but not in the cerebellum, wherein the CaMKIl $\alpha$ gene is not expressed (Figure $2 \mathrm{e}$ ). TAK1 $1^{\text {nKO }}$ mice did not show any obvious behavioral phenotype; nor did we observe any spontaneous neuronal cell death or morphological change (data not shown). Interestingly, deletion of TAK1 in neurons of the cortex did not reduce the infarct size (Figure 2f). Taken together, these results demonstrate that acute, but not chronic inhibition or deletion of TAK1 protects neurons during cerebral ischemia.

TAK1 inhibition has a transient effect on JNK and p38/ MAPK activity. TAK1 is a central activator of NF- $\kappa \mathrm{B}$, JNK, and p38/MAPK signaling. To elucidate the effect of TAK1 inhibition on these downstream signaling pathways, we treated cortical neurons with the solvent DMSO or with OZ for either 10 days (chronic) or $1 \mathrm{~h}$ (acute inhibition) before OGD. Whole-cell extracts were prepared at 0,5 , or $30 \mathrm{~min}$ after OGD. OGD activated NF- $\kappa \mathrm{B}$, as shown by a transient degradation of $\mathrm{I} \kappa \mathrm{B} \alpha(0 \mathrm{~min}$ after OGD, Figure 3a). As expected, NF- $\kappa \mathrm{B}$ activation was prevented if neurons were pretreated for 10 days or for $1 \mathrm{~h}$ with $\mathrm{OZ}$ (Figure 3a). Activation of $\mathrm{p} 38 / \mathrm{MAPK}$ by OGD persisted for at least $30 \mathrm{~min}$ after OGD. Interestingly, p38/MAPK activation by OGD was not affected by pretreatment with $\mathrm{OZ}$ for 10 days, whereas only transient p38/MAPK activation was observed in the presence of acute TAK1 inhibition. As shown in Figure 3b, 
a

$$
\begin{gathered}
+\mathrm{TNF} \\
+\mathrm{OZ}
\end{gathered}+\mathrm{TNF}+0^{\prime}+5^{\prime}+30^{\prime}
$$

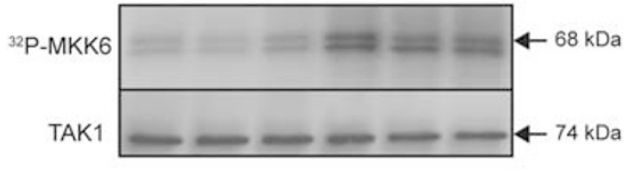

b
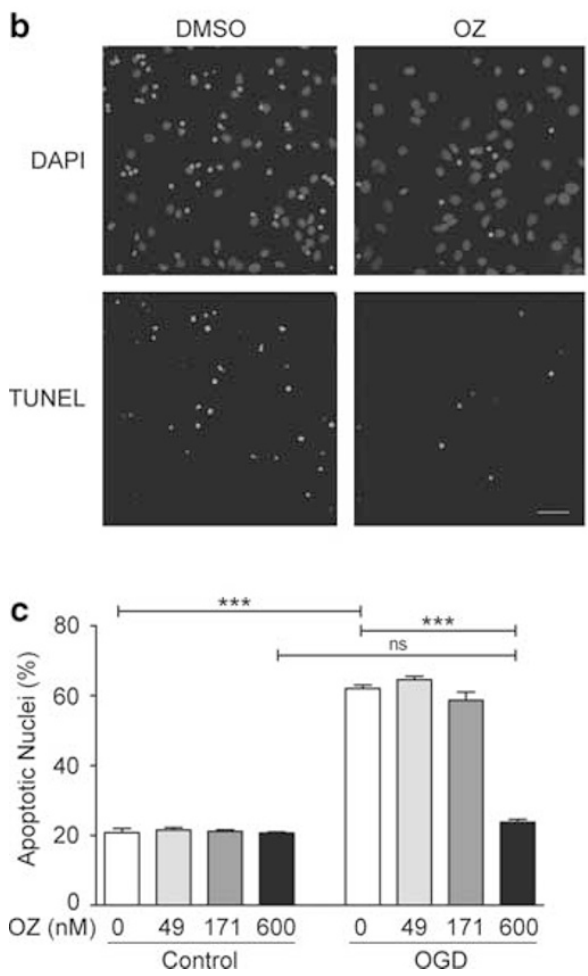

d

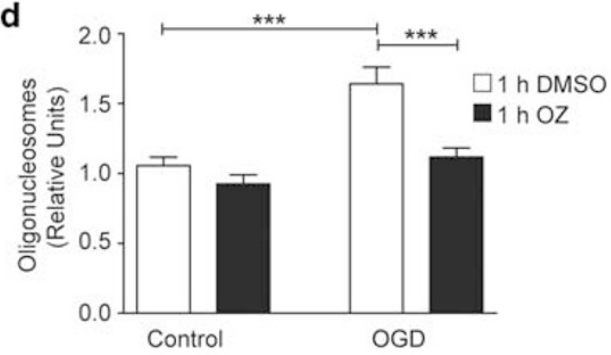

e
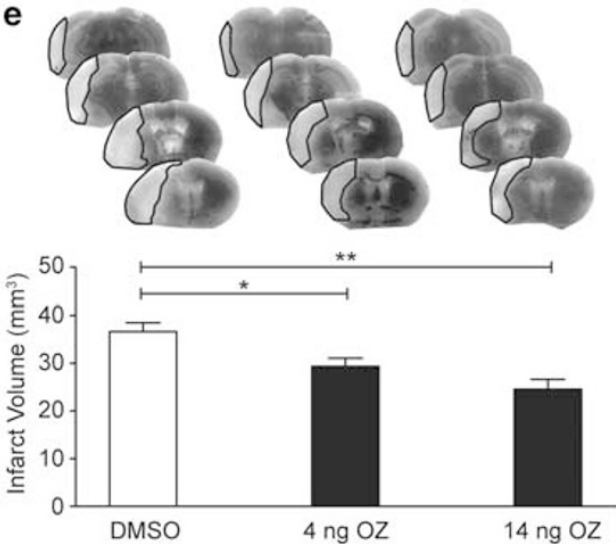

f
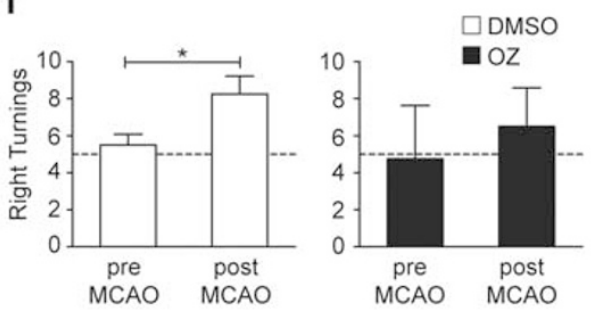

Figure 1 Acute inhibition of TAK1 during oxygen glucose deprivation (OGD) and middle cerebral artery occlusion (MCAO) is neuroprotective. (a) TAK1 activity was monitored by a pull-down kinase assay. Primary cortical neurons were either stimulated with TNF (10 ng/ml), preincubated with $5 Z-7-0 x 0 z$ eaenol $(O Z, 600 \mathrm{nM})$, or subjected to 4.5-h OGD and the indicated recovery times. Equal precipitation of TAK1 was verified by immunoblotting of the same membrane. A typical example of four independent experiments is shown. C, control. (b) TUNEL and DAPI staining of primary cortical neurons after 4.5-h OGD and a 24-h recovery period. Scale bar: $20 \mu \mathrm{m}$. (c) Quantification of apoptotic nuclei in neurons after 4.5-h OGD and a 24-h recovery period. Neurons were treated with the indicated concentrations of OZ for $1 \mathrm{~h}$ before OGD. Nuclei were stained by DAPI. Values are means \pm S.E.M., $N=3$. One-way ANOVA: $F(7 / 16)=324.5, P<0.0001$. ${ }^{* \star *} P<0.0001$ (Newman-Keuls post hoc test). ns, not significant. (d) Quantification of the amount of oligonucleosomes in the cytoplasm of neurons as a sign of DNA fragmentation after 4.5-h OGD and a 24-h recovery. Values are means \pm S.E.M., $N=10$. One-way ANOVA: $F(3 / 39)=15.03, P<0.0001$, ${ }^{* \star *} P<0.001$ (Newman-Keuls post hoc test). (e and f) Mice were subjected to MCAO after intracerebroventricular injections of $\mathrm{OZ}$. (e) Upper panel, silver-stained brain sections with labeled infarct area $48 \mathrm{~h}$ after MCAO; lower panel, evaluation of infarct volume $48 \mathrm{~h}$ after MCAO. Values are means \pm S.E.M., $N=5-6$, one-way ANOVA: $\mathrm{F}(2 / 22)=9.071, P=0.0016,{ }^{*} P<0.01,{ }^{* \star} P<0.001$ (Newman-Keuls post hoc test). (f) Mice were tested for sensorimotor function by corner tests before and $24 \mathrm{~h}$ after MCAO. A total of 10 turnings were evaluated per mouse. Values are means \pm S.E.M., $N=5-6$. ${ }^{*} P=0.0027$ (Student's $t$-test)

phosphorylation of p38/MAPK was markedly reduced $30 \mathrm{~min}$ after OGD by 1 -h OZ treatment. OGD also activated JNK signaling as demonstrated by the increased phosphorylation of the substrate c-Jun (Figure 3c). In neurons pretreated chronically with OZ, c-Jun was phosphorylated for at least 30 min after OGD, whereas no activation was seen in neurons pretreated acutely with OZ (Figure 3c). These data show that both acute and chronic inhibition of TAK1 block $\mathrm{NF}-\kappa \mathrm{B}$ activation but their effects on JNK and p38/MAPK signaling differ. Thus, blocking JNK and/or p38/MAPK might be responsible for the protective effect of acute TAK1 inhibition.
In support of this view SB203580, a specific inhibitor of p38/MAPK, and D-JNKI, a JNK inhibitor, blocked cell death induced by OGD (Figure 3d). Interestingly, the protective effect of both compounds was preserved if cells were pretreated with $\mathrm{OZ}$ for 10 days (Figure 3d), demonstrating that after prolonged TAK1 inhibition p38/MAPK and JNK are still involved in cell death but are activated independent from TAK1.

To test whether TAK1 inhibition would also interfere with activity of the MAP kinases p38/MAPK and JNK in vivo, we treated mice with $\mathrm{OZ}$ (14 ng i.c.v.) at $20 \mathrm{~min}$ before MCAO, and evaluated the phosphorylation of p38/MAPK and c-Jun 

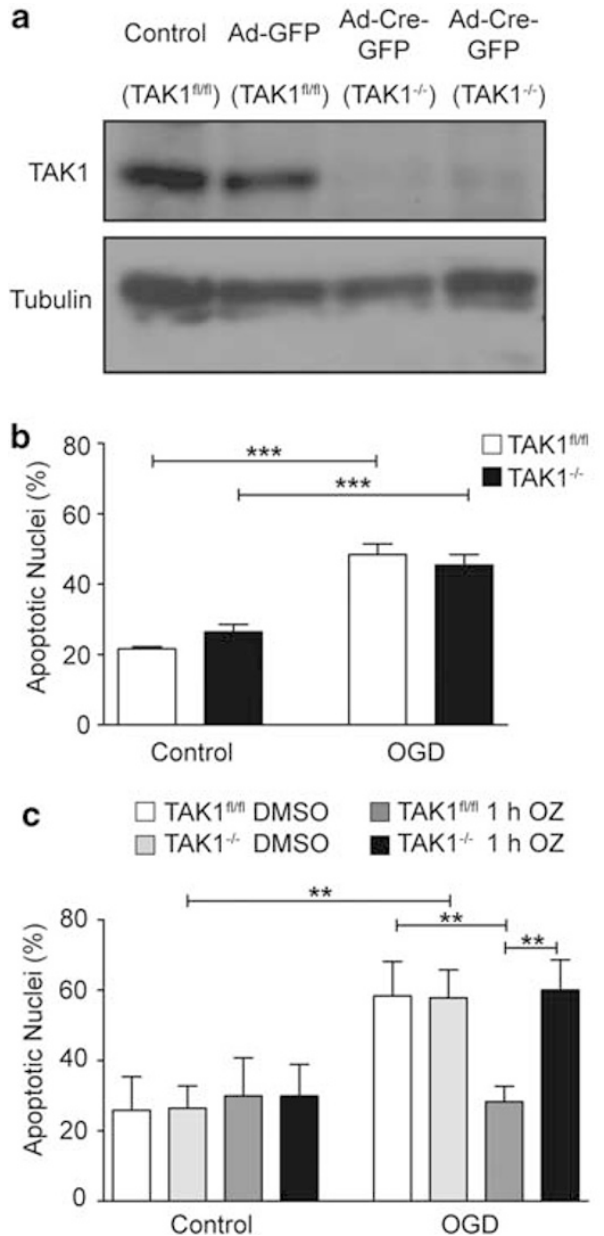

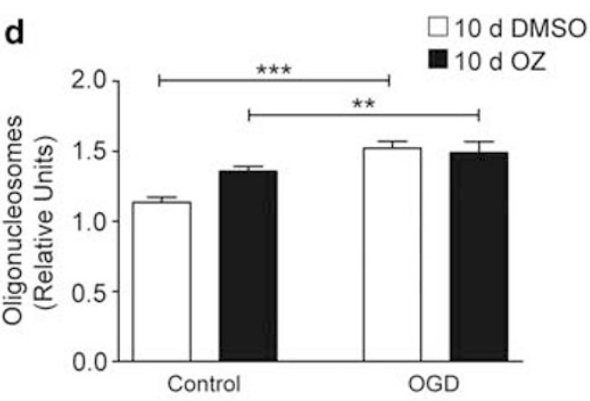

e

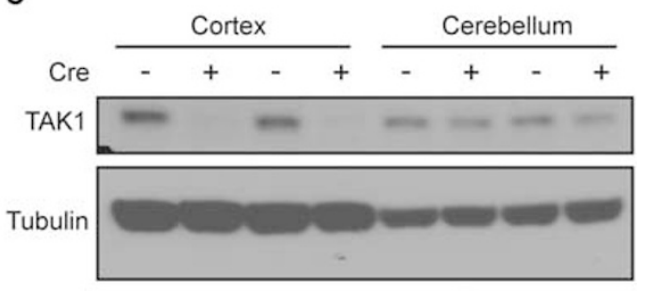

f

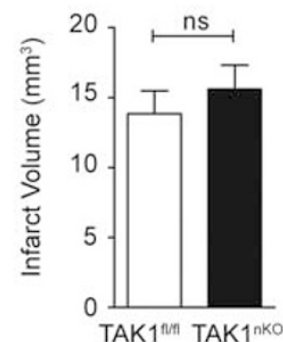

Figure 2 Acute, but not chronic inhibition or deletion of TAK1 is neuroprotective in ischemia. (a) Immunoblotting of TAK1 in primary cortical neurons at 9 days after infection of TAK $1^{\text {fl/fl }}$ neurons with Ad-GFP or Ad-Cre-GFP. The control group was not infected. Tubulin was used to control protein loading in each lane. (b and $\mathbf{c}$ ) Quantification of apoptotic nuclei of TAK1 ${ }^{\text {fl/til }}$ and TAK1 ${ }^{-1-}$ primary cortical neurons after 4.5-h OGD and 24-h recovery. (b) Values are means \pm S.E.M., $N=4$, one-way ANOVA: $F(3 /$ $15)=11.87, P=0.0007,{ }^{* \star *} P<0.0001$ (Newman-Keuls post hoc test). (c) The protective effect of OZ depends on TAK1. Neurons were pretreated for $1 \mathrm{~h}$ with either $600 \mathrm{nM}$ $\mathrm{OZ}$ or DMSO. Values are means \pm S.E.M., $\quad N=3$, one-way ANOVA: $F(7 / 41)=22.74, \quad P<0.0001,{ }^{* \star} P<0.001$ (Newman-Keuls post hoc test). (d) Quantification of the amount of oligonucleosomes in the cytoplasm of neurons after 10 days of pretreatment with $600 \mathrm{nM}$ OZ or DMSO, 4.5-h OGD, and 24-h recovery. Values are means \pm S.E.M., $N=10$, one-way ANOVA: $F(3 / 39)=11.64, P<0.0001,{ }^{* \star} P<0.0001,{ }^{* \star} P<0.001$ (Newman-Keuls post hoc test). (e) Evaluation of TAK1 in protein lysates from cortex and cerebellum of CaMKIl $\alpha-\mathrm{CreER}^{\mathrm{T} 2}$-positive (TAK1 ${ }^{\mathrm{nKO}}$ ) or -negative (TAK1 $1^{\text {fl/fll }}$ ) mice by immunoblotting. Tubulin was used to control loading of protein in each lane. (f) Evaluation of infarct volume of TAK $1^{\text {nKO }}$ and control mice. Values are means \pm S.E.M., $N=16-17$. ns, not significant

$60 \mathrm{~min}$ after MCAO in cortical lysates. TAK1 inhibition significantly reduced the activity of p38/MAPK after MCAO but had no effect on c-Jun phosphorylation (Figure 3e). A similar finding was obtained at $180 \mathrm{~min}$ after MCAO (data not shown). These data confirm in vivo that TAK1 mediates the activation of p38/MAPK by cerebral ischemia. The reason for the discrepancy between in vivo and in vitro data on the activation of JNK is unclear, but could lie in the fact that neuronal activation of JNK by TAK1 is concealed by effects in other cell types of the tissue extracts.

Acute TAK1 inhibition reverses the induction of Cox-2, Nox-2, and $p 40^{\text {phox }}$ by OGD. Activation of p38/MAPK and c-Jun was impaired after OGD in neurons pretreated acutely, but not chronically with OZ. Next, we examined the expression of the target genes Cox-2 (Ptgs2), Nox-2, and $p 40^{\text {phox }}{ }^{27-29}$ In keeping with our results, we found that
Cox-2, Nox-2, and $p 40^{\text {phox }}$ were induced at $3 \mathrm{~h}$ after OGD, but induction was impaired in neurons after acute inhibition of TAK1 (Figures 4a-c). However, chronic inhibition of TAK1 did not interfere with the upregulation of Cox-2 and Nox-2, and only partially reduced the induction of $p 40^{\text {phox }}$ by OGD in neurons (Figures $4 \mathrm{~d}-\mathrm{f}$ ). As the Nox-2 and the $p 40^{\text {phox }}$ genes encode for subunits of the NADPH oxidase, an enzyme that is responsible for superoxide formation, and COX-2 produces superoxide, too, we examined the level of superoxide production in neurons after OGD by incubating cortical neurons with dihydroethidine (DHE). By quantifying DHE-positive nuclei we found that acute, but not chronic, inhibition of TAK1 decreased the formation of superoxide anions after OGD (Figure 5a). Next, we measured levels of reduced glutathione (GSH), a superoxide scavenger and anti-apoptotic factor in the brain. ${ }^{30} \mathrm{GSH}$ levels were reduced after OGD, but less so if neurons were pretreated for $1 \mathrm{~h}$ with 
a

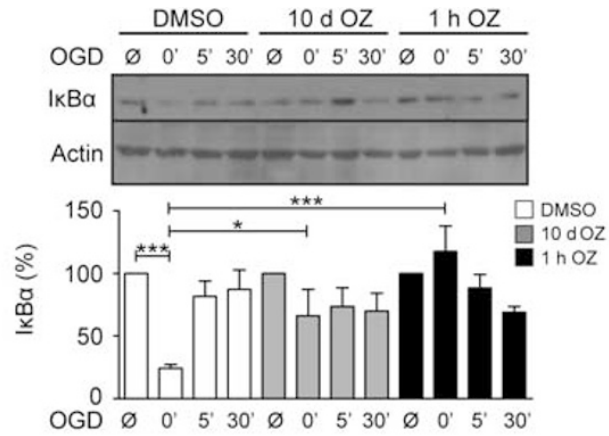

C
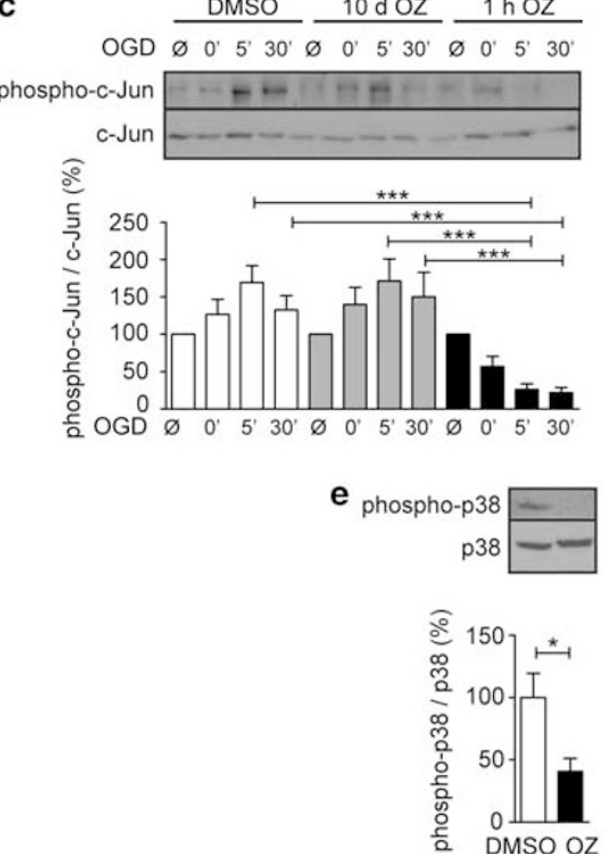

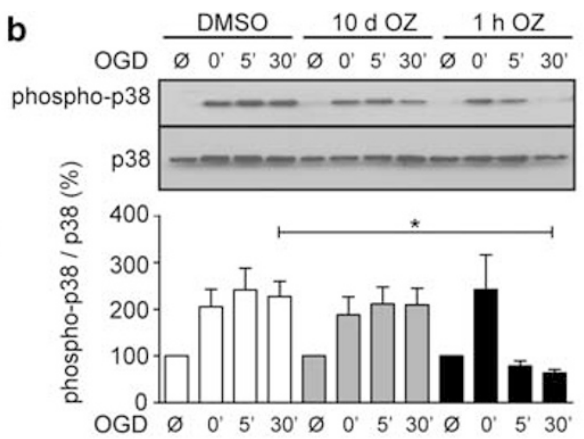

d

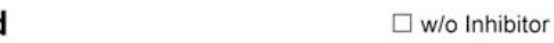

$\square$ p38 Inhibitor

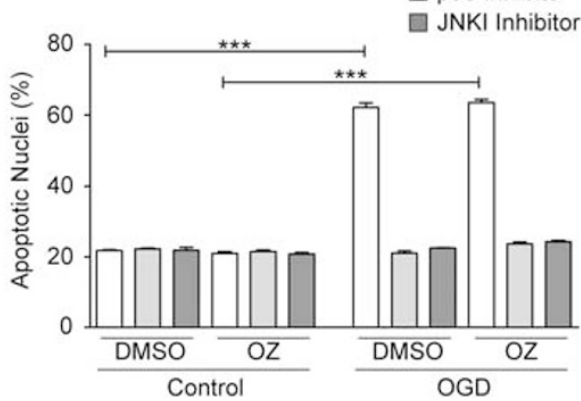

phospho-c-Jun $=$

Figure 3 Transient effect of TAK1 inhibition on p38/MAPK and JNK but not on NF- $\kappa \mathrm{B}$ signaling in vitro. (a-c) After incubation for 10 days or $1 \mathrm{~h}$ with $\mathrm{OZ}$ (600 nM) or DMSO, neurons were exposed to OGD followed by increasing recovery times. Controls received no OGD ( $)$. Protein lysates were analyzed by immunoblotting to determine the degradation of $I_{\kappa} \mathrm{B} \alpha$, a marker of NF- $\kappa \mathrm{B}$ activation, and the phosphorylation of p38/MAPK and c-Jun. Values are means \pm S.E.M., of four independent experiments. ${ }^{* \star *} P<0.0001,{ }^{*} P<0.01$ (Newman-Keuls post hoc test). (d) Quantification of apoptotic nuclei after 4.5-h OGD and 24 -h recovery. $0 Z$ ( $600 \mathrm{nM}$ ) was added at 10 days before OGD, the p38 inhibitor SB203580 $(15 \mu \mathrm{M})$ and the JNK inhibitor D-JNKI $(10 \mu \mathrm{M}) 1 \mathrm{~h}$ before OGD as indicated. One-way ANOVA: $\mathrm{F}(11 / 35)=644.0, P<0.0001,{ }^{* \star \star} P<0.0001$ (Newman-Keuls post hoc test). (e) Immunoblotting of (phospho)-p38/MAPK and (phospho)-c-Jun in lysates of the ischemic cortex 60 min after mice were treated with $0 Z$ (14 ng, i.c.v.) or DMSO and subjected to MCAO. Lower panel, quantification of five to six independent experiments. Values are means \pm S.E.M. ${ }^{\star} P<0.05$ (Student's $t$-test)

OZ. In contrast, no difference in GSH levels was found in ischemic neurons after chronic inhibition of TAK1 (Figure 5b). These results suggest that the reduced formation of superoxide anions in neurons after acute inhibition of TAK1 mediates neuroprotection.

Upregulation of ASK-1 compensates for chronic TAK1 inhibition. To investigate the discrepancy that acute, but not chronic, inhibition of TAK1 provides neuroprotection in ischemia, we sought to determine whether other MAP3 kinases might compensate for TAK1 activation and mediate selective induction of JNK and/or p38/MAPK, but not NF- $\kappa B$ after OGD. Therefore, we performed RT-PCR on total RNA from neuronal cultures incubated for either 10 days or $1 \mathrm{~h}$ with OZ. We found that Ask-1 was significantly induced in neurons with chronic, but not acute, inhibition of TAK1 (Figures $6 \mathrm{a}$ and b). However, we could not detect any changes in the expression of the following MAP3 kinases: Mlk2, Mlk3, Mekk1, Mekk3, Mekk4, Dlk, or Tao2 (Figures 6a-c, data not shown). To determine whether induction of Ask-1 underlies the lack of efficacy of chronic TAK1 inhibition, we blocked ASK-1 activity by viral expression of a dominant negative form of ASK-1, ASK-1-KM. ${ }^{31}$ As reported previously inhibition of ASK-1 reduced neuronal cell death in response to OGD (Figure 6d). ${ }^{32}$ Importantly, when ASK-1 was inhibited prolonged $\mathrm{OZ}$ treatment further improved cell survival after OGD. This is in accordance with the notion that upregulation of ASK-1 underlies the lack of efficacy of chronic TAK1 inhibition. In line with the known pro-apoptotic function of ASK $-1,{ }^{33}$ we found that chronic inhibition of TAK1 by OZ induced a significant increase in apoptotic nuclei (Figure 6e). This effect was confounded in other experiments (Figure 2c) by a higher rate of apoptosis that was because of the additional medium changes to control for OGD. 

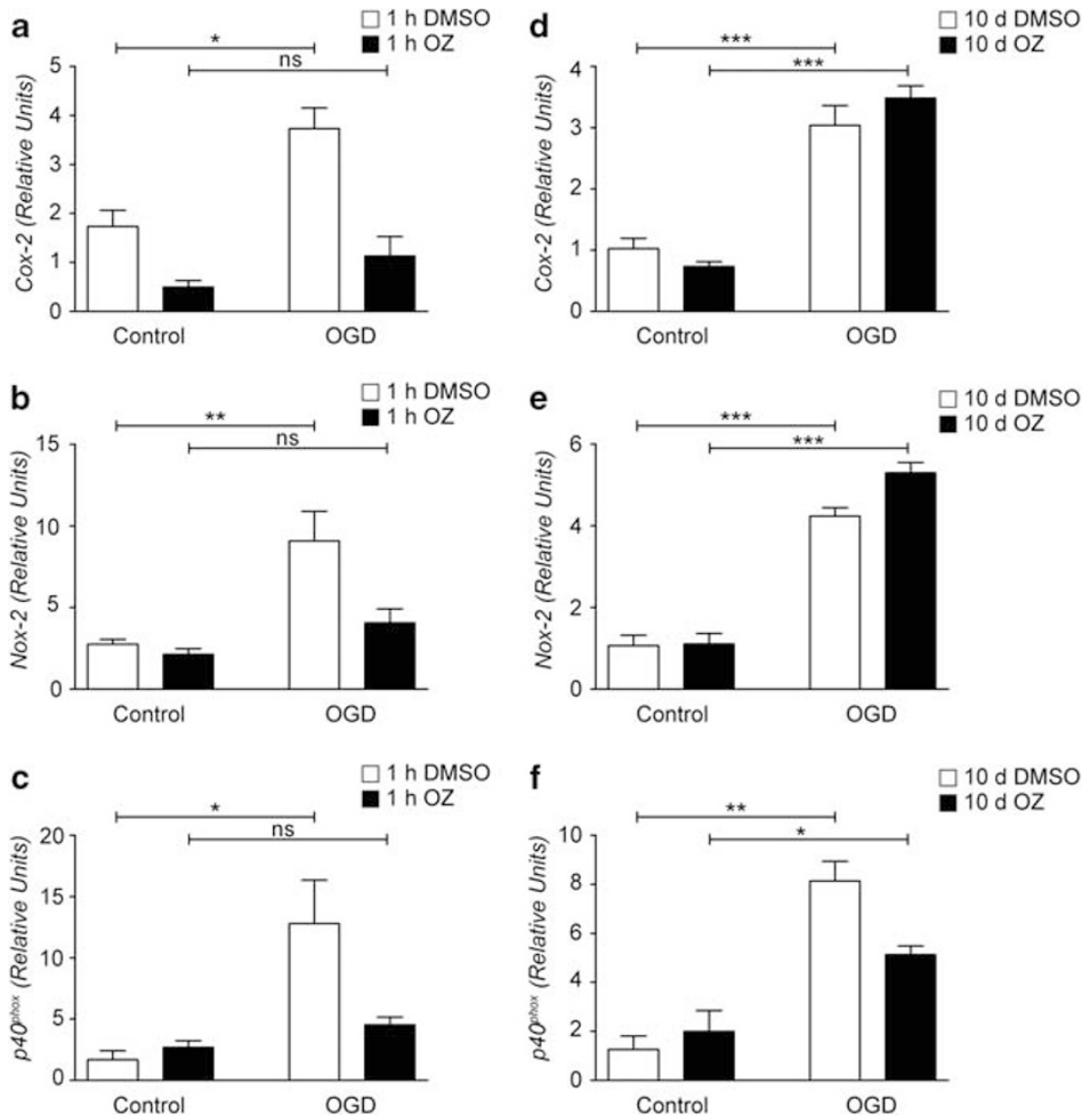

Figure 4 Acute TAK1 inhibition reduces expression of Cox-2, Nox-2, and p40 $0^{\text {phox }}$ after OGD. (a-f) mRNA of Cox-2, Nox-2, and p40 phox was quantified by RT-PCR in cortical neurons at $3 \mathrm{~h}$ after 4.5-h OGD. (a-c) Neurons were pretreated for $1 \mathrm{~h}$ with $\mathrm{OZ}$ (600 nM) or DMSO; values are means \pm S.E.M., N=2-3, Cox-2: one-way ANOVA: $F(5 / 13)=5.642, P=0.0016,{ }^{*} P<0.01$ (Newman-Keuls post hoc test), Nox-2: one-way ANOVA: $F(3 / 9)=14.19, P=0.0039,{ }^{* *} P<0.001$ (Newman-Keuls post hoc test), p40 $0^{\text {phox: }}$ one-way ANOVA: $F(5 / 13)=5.564, P=0.017$, ${ }^{\star} P<0.01$ (Newman-Keuls post hoc test). ns, not significant. (d-f) Neurons were pretreated for 10 days with $0 Z$ $(600 \mathrm{nM})$ or DMSO; values are means \pm S.E.M., $N=2-3$, Cox-2: one-way ANOVA: $F(3 / 9)=56.5, P<0.0001,{ }^{\star \star \star} P<0.0001$ (Newman-Keuls post hoc test), Nox-2: one-way ANOVA: $F(3 / 9)=67.55, P<0.0001,{ }^{* * *} P<0.0001$ (Newman-Keuls post hoc test), $p 40^{\text {phox: }}$ one-way ANOVA: $F(3 / 9)=18.33, P=0.002,{ }^{* *} P<0.001$, ${ }^{*} P<0.01$ (NewmanKeuls post hoc test)

In summary, we propose that acute TAK1 inhibition exerts its neuroprotective effect in OGD by blocking JNK and p38/ MAPK activation, and thereby interfering with the upregulation of pro-oxidative genes such as Nox-2, p40 phox, and Cox-2, and the subsequent increase in superoxide formation. Chronic inhibition or deletion, in contrast, is compensated by upregulation of ASK-1 that activates JNK and p38/MAPK (Figure 7).

\section{Discussion}

The data presented in this study demonstrate that ischemia activates the MAP3K TAK1 in neurons. In line with current knowledge that TAK1 is an upstream activator of several signaling pathways, including JNK, p38/MAPK, and NF- $\kappa \mathrm{B}$, we found that activation of these pathways in neurons by OGD is mediated by TAK1. Accordingly, the induction of Nox-2 $\left(\right.$ gp91 $\left.1^{\text {phox }}\right), p 40^{\text {phox }}$, and Cox-2, target genes of AP-1 and NF- $\kappa \mathrm{B},{ }^{27-29}$ depends on TAK1 activity. Upregulation of COX-2 and the NADPH oxidase with its key subunits NOX-2 and $\mathrm{p} 40^{\text {phox }}$ are considered to be important sources of superoxide anions in cerebral ischemia. ${ }^{34-36}$ This might be the mechanism by which TAK1 promotes oxidative stress in ischemic neurons. Consistent with the central role of superoxide anions in cerebral ischemia, both NOX-2- and COX-2-deficient mice are protected against cerebral ischemia. ${ }^{37,38}$ Thus, blocking the induction of Nox-2, p40 phox and Cox-2 as well as superoxide production may explain how acute inhibition of TAK1 protects against cerebral ischemia both in vitro and in vivo.

In our experiments, TAK1 inhibition involves the smallmolecule inhibitor OZ. This compound inhibits TAK1 but not other MAP3Ks. ${ }^{23}$ The specificity of $\mathrm{OZ}$ is confirmed by the finding that $\mathrm{OZ}$ lost its efficacy in $\mathrm{TAK} 1^{-1-}$ neurons. Therefore, it came as a surprise that genetic deletion of TAK1 did not confer protection against cerebral ischemia. Likewise, longterm treatment with $\mathrm{OZ}$ to mimic the protracted time course of genetic deletion had no effect on neuronal survival after ischemia. The transience of $\mathrm{OZ}$ effects suggests that other mechanisms compensate for long-term inhibition or deficiency of TAK1. Indeed, after prolonged TAK1 inhibition, we found an upregulation of the MAP3K Ask-1. How TAK1 
a

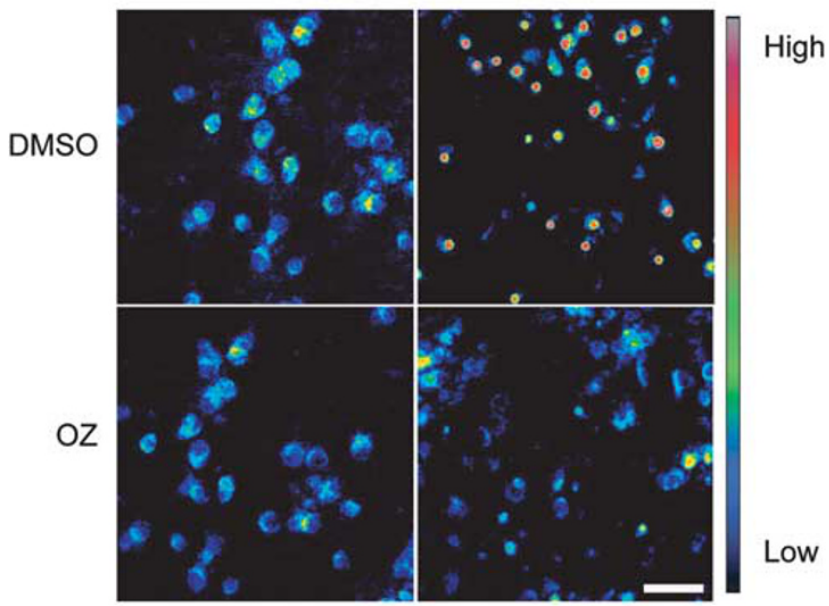

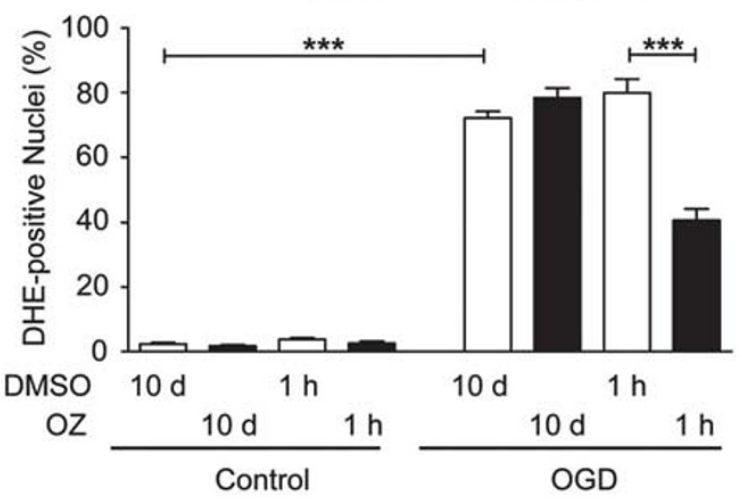

b

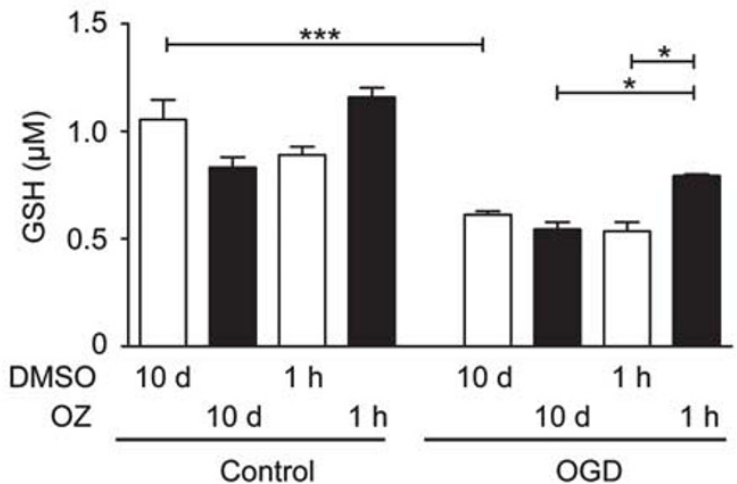

Figure 5 Acute TAK1 inhibition reduces superoxide formation and increases reduced glutathione after OGD. (a) Neurons were incubated with dihydroethidium $(\mathrm{DHE}, 5 \mu \mathrm{M})$ for $30 \mathrm{~min}$ after 4.5-h OGD. Production of superoxide anions was analyzed by quantifying DHE-positive nuclei. False color images were generated according to fluorescence intensity using ImageJ software, scale bar: $20 \mu \mathrm{m}$ (upper panel). Values are means \pm S.E.M., $N=6$, one-way ANOVA: $F(7 / 15)=310.8$, $P<0.0001,{ }^{* * *} P<0.0001$ (Newman-Keuls post hoc test). (b) Levels of reduced glutathione (GSH) were quantified at $30 \mathrm{~min}$ after 4.5 -h OGD. Neurons were treated with $\mathrm{OZ}(600 \mathrm{~nm})$ for 10 days (d) or $1 \mathrm{~h}$. Values are means \pm S.E.M., $N=3$, oneway ANOVA: $F(7 / 23)=24.34, P<0.0001,{ }^{* \star} P<0.0001$, ${ }^{\star} P<0.01$ (NewmanKeuls post hoc test)

inhibition stimulates Ask-1 expression is unclear so far. A possible explanation involves E2F, the major transcriptional regulator of $A s k-1 .{ }^{33}$ As E2F is blocked by NF- $\kappa$ B signaling, ${ }^{39}$ inhibition of TAK1 and subsequently of NF- $\kappa$ B may release E2F to stimulate Ask-1 expression. A dominant-negative mutant of ASK-1 restored the neuroprotective effect of prolonged TAK1 inhibition, demonstrating that ASK-1 mediates the compensatory mechanism that leads to inefficacy of prolonged TAK1 inhibition. Upregulation of ASK-1 is known to induce apoptosis, ${ }^{33}$ and this may be why we observed a slightly enhanced baseline rate of apoptosis during prolonged TAK1 inhibition in vitro (Figure 6e). However, in TAK $1^{\text {nKO }}$ mice there was no evidence of neuronal apoptosis or neuronal cell loss in cortex and hippocampus (data not shown), suggesting that prolonged TAK1 inhibition is unlikely to lead to neurotoxic effects in vivo.

ASK-1 activates p38/MAPK and JNK but not NF- $\kappa$ B signaling, ${ }^{33}$ explaining why $\mathrm{NF}-\kappa \mathrm{B}$ inhibition persisted, but the blockade of p38/MAPK and JNK was only transient after $\mathrm{OZ}$ treatment (Figure 3). After OGD, the effects of TAK1 inhibition on neuronal cell death paralleled its effects on p38/ MAPK and JNK signaling, suggesting that inhibition of these two pathways is responsible for the neurotoxic effect of TAK1 activation in vitro. However, whether neuronal JNK is really activated by TAK1 during cerebral ischemia in vivo is questionable (Figure $3 e$ ) and has to be investigated in future studies. The inhibition of NF- $\kappa \mathrm{B}$ signaling that persisted with prolonged TAK1 inhibition was not able to protect neurons against ischemia, in contrast to the protection afforded by a selective NF- $\kappa$ B inhibition. ${ }^{6}$ This discrepancy may be related to a lower apoptotic threshold due to increased ASK-1 levels after prolonged TAK1 inhibition. Similar to our data, TAK1 deficiency in the liver was shown to activate the MAP3 kinase TAO2. ${ }^{21}$ Thus, MAP3 kinases seem to form a plastic signaling network that readily adjusts to inhibition of an individual kinase. These adaptive processes will clearly affect the interpretation of genetic knockout studies.

Our data suggest that long-term TAK1 inhibition may not be protective in neurodegenerative disorders. Moreover, TAK1 deficiency caused apoptosis in keratinocytes, hepatocytes, and hematopoietic cells, raising serious safety concerns with respect to chronic TAK1 inhibition. ${ }^{19,21,40}$ However, the situation is different for treating acute stroke. A single dose of a specific TAK1 inhibitor was neuroprotective by blocking a key step in inflammatory pathways of ischemic brain damage. The short-term administration of a TAK1 inhibitor required for treatment of acute stroke is unlikely to cause toxicity of other organs.

\section{Materials and Methods}

Materials. OZ (MW, $362.4 \mathrm{~g} / \mathrm{mol}$ ) was kindly provided by Dr. K Takehana (Ajinomoto Co., Kawasaki, Japan,) and was purchased from AnalytiCon Discovery GmbH (Potsdam, Germany).

Anti-rabbit polyclonal antibody was purchased from Santa Cruz Biotechnology (Santa Cruz, CA, USA). Rabbit antibodies against phosphorylated and nonphosphorylated forms of p38/MAPK and c-Jun were purchased from Cell Signaling Technology (Beverly, MA, USA). Antibody against total TAK1 was purchased from Upstate (Charlottesville, USA, USA). To generate an anti-TAK1 antibody that recognizes exon 2, we immunized rabbits with the following peptide: RAKDVAIKQIESESERK. The antibody was purified by affinity chromatography. Ad-Cre-GFP was purchased from Vector Biolabs (Philadelphia, PA, USA); Ad-ASK-1-KM was purchased from Seven Hills Bioreagents (Cincinnati, OH, USA); Ad-GFP was kindly provided by Dr. D Nettelbeck (Heidelberg, Germany). 
a

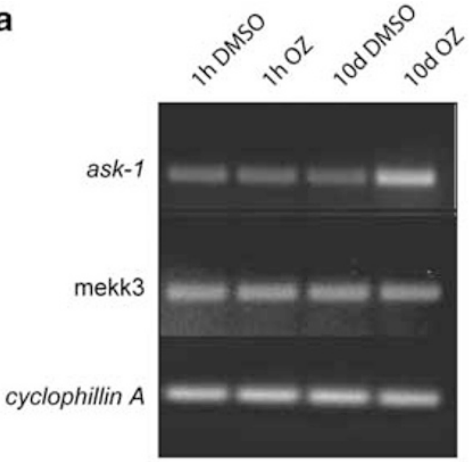

b

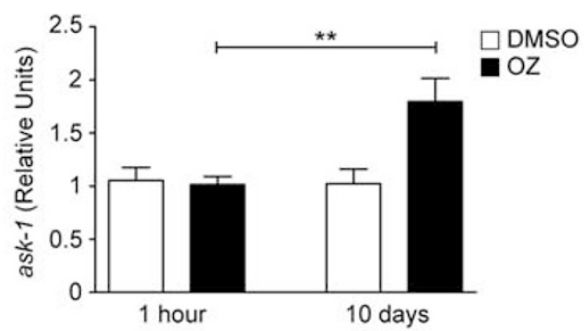

C

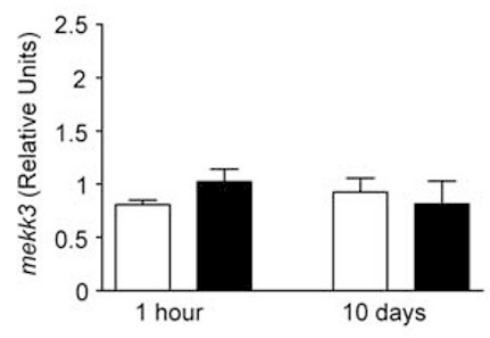

d

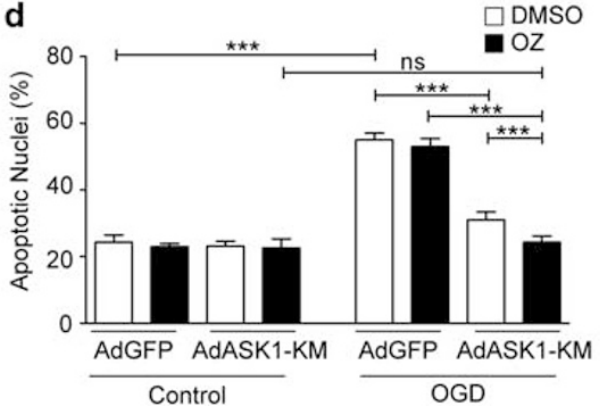

e

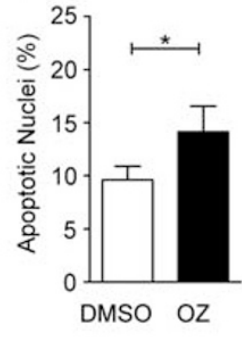

Figure 6 Upregulation of ASK-1 compensates for TAK1 activity on long-term inhibition. (a) RT-PCR demonstrated upregulation of Ask-1 but not Mekk3 after prolonged TAK1 inhibition. (b and $\mathbf{c})$ Quantification of mRNA expression of $A s k-1$ and Mekk3 by RT-PCR. Ask-1: values are means \pm S.E.M., $N=6$, one-way ANOVA: F(3/22) $=6.694$, $P=0.0029,{ }^{*} P<0.001$ (Newman-Keuls post hoc test). Mekk3: values are means \pm S.E.M., $N=4$, one-way ANOVA: $F(3 / 15)=0.5238, P=0.6741$. (d) Quantification of apoptotic nuclei after viral expression of the dominant-negative ASK-1 inhibitor ASK-1-KM, treatment with OZ (600 nM) for 10 days, and exposure to 4.5-h OGD and 24-h recovery as indicated. Primary cortical neurons were infected on DIV 8 and exposed to OGD on DIV 10. Values are means \pm S.E.M., $N=4$, one-way ANOVA: $F(7 / 31)=177.2, P<0.0001$. ${ }^{* \star} P<0.0001$ (Newman-Keuls post hoc test). ns, not significant. (e) Quantification of apoptotic nuclei after inhibition of TAK1 by OZ (600 nM) for 10 days in vitro. In this experiment, there was no additional medium change. Values are means \pm S.E.M, $N=3,{ }^{*} P=0.0152$ (Student's $t$-test)

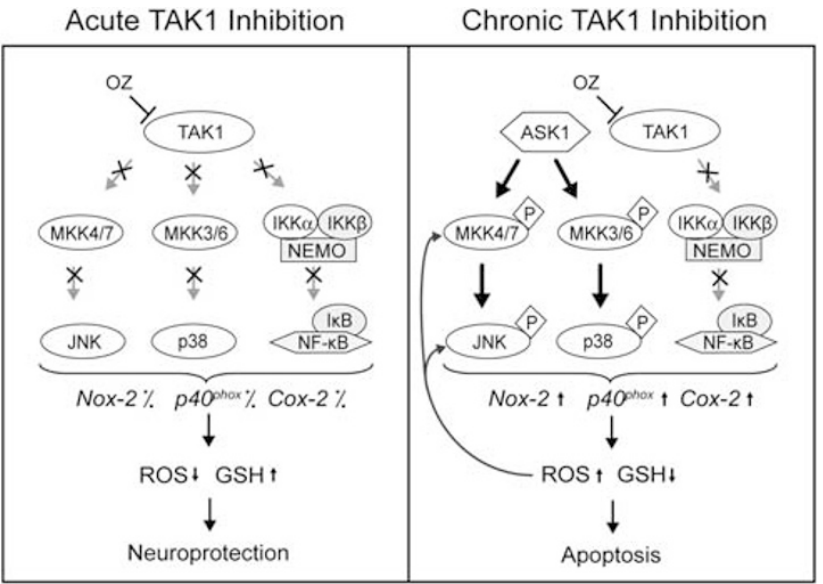

Figure 7 Proposed mechanism by which short-term TAK1 inhibition protects neurons against ischemia. Acute TAK1 inhibition by OZ blocks activation of JNK, p38/MAPK, and NF- $\kappa \mathrm{B}$, and thereby provides neuroprotection. Chronic TAK1 inhibition induces expression of ASK-1 that compensates for TAK1 inhibition with respect to JNK and p38/MAPK activation, transcription of Cox-2, p40 ${ }^{\text {ohox }}$, and Nox-2 and formation of reactive oxygen species, paving the way to cell death

Cell culture and virus infection. NMRI mice were used to generate wildtype cultures and TAK $1^{\text {fl/til }}$ mice for TAK $1^{-1-}$ cultures. Primary cortical neurons were prepared from mouse embryos at embryonic day 16 (E16) as described previously. ${ }^{9}$ In brief, cerebral cortices were dissected and freed from the meninges, hippocampus, and olfactory bulb and incubated in pre-warmed trypsin-EDTA for $8 \mathrm{~min}$ at $37^{\circ} \mathrm{C}$. After washing with prewarmed PBS, cell clusters were resupended in $3 \mathrm{ml}$ of full neurobasal medium (supplemented with $2 \%$ B27, 0.5 mM L-glutamine, $100 \mathrm{IU} / \mathrm{ml}$ penicillin, and $100 \mu \mathrm{g} / \mathrm{ml}$ streptomycin) and separated into single cells by triturating through a fire-polished Pasteur pipette. Then, $25 \times 10^{4}$ or $10^{6}$ cells were plated per well of a 24- or 6-well plate, coated with poly-D-lysine. The cells were incubated at $37^{\circ} \mathrm{C}$ with $5 \% \mathrm{CO}_{2}$. The next day, half volume of medium was exchanged by neurobasal medium supplemented with $5 \%$ FCS. Then half volume of medium was exchanged each third day by full neurobasal medium. Experiments were performed on day in vitro (DIV) 10 . We previously found that this procedure produces cultures in which $>95 \%$ of cells are positive for the neuronal marker NeuN. ${ }^{9}$ For adenoviral transduction of neurons, three MOI of Ad-Cre-GFP or Ad-GFP were added to $10^{6}$ cells on DIV 1 and incubated for 2 days. Then, medium was exchanged every 2 days. For transduction with Ad-ASK-1-KM or Ad-GFP control virus, neurons were infected with three MOI on DIV 8.

Oxygen glucose deprivation. OGD was used as an in vitro model of ischemia. Primary cortical neurons were used on DIV 10. Medium was exchanged with full neurobasal medium containing OZ or DMSO. After $1 \mathrm{~h}$, half volume of medium was exchanged with neurobasal medium containing 2-deoxy-D-glucose ( $5 \mathrm{mM}$ end concentration) and incubated for $1 \mathrm{~h}$. Cells were transferred to an anaerobic chamber that was flushed with $\mathrm{CO}_{2} / \mathrm{N}_{2}(5 \% / 95 \%)$ for $15 \mathrm{~min}$. The chamber was tightly sealed and cells were incubated at $37^{\circ} \mathrm{C}$ for $4.5 \mathrm{~h}$. Subsequently, medium was replaced by fresh medium.

Detection and quantification of apoptosis. Primary cortical neurons on coverslips were stained after OGD using TUNEL (In Situ Cell Death Detection Kit, Roche Applied Science, Penzberg, Germany). After OGD, cells were fixed with $4 \%$ PFA for $20 \mathrm{~min}$ and permeabilized for $5 \mathrm{~min}$ at $4^{\circ} \mathrm{C}$ in PBS containing $0.1 \%$ triton $X-100$. Then, cells were incubated with TUNEL reaction mix for $1 \mathrm{~h}$ at room temperature in the dark and stained with DAPI (Roth, Karlsruhe, Germany) for 
5 min. We counted neurons with TUNEL staining or with condensed chromatin in five randomly selected fields and expressed the number of apoptotic neurons relative to the total cell count.

Cell-death ELISA ${ }^{\text {PLUS }}$ (Roche Applied Science) was performed according to the manufacturer's protocol.

Middle cerebral artery occlusion. Mice were anesthetized by intraperitoneal injection of $150 \mu \mathrm{l} 2.0 \%$ tribromoethanol per $10 \mathrm{~g}$ body weight. A skin incision was made between the ear and the orbit on the left side. The temporal muscle was removed by electrical coagulation. The stem of the MCA was exposed through a burr hole and occluded by microbipolar coagulation (Erbe, Tubingen, Germany). Surgery was performed under a microscope (Hund, Wetzlar, Germany). At $20 \mathrm{~min}$ before MCAO, we injected vehicle (DMSO) or OZ solution (both $2 \mu \mathrm{l}$ ) into the lateral ventricle, using a $10-\mu \mathrm{l} \mathrm{Hamilton}$ syringe. ${ }^{6}$ During the whole procedure, a body temperature of $37^{\circ} \mathrm{C}$ was maintained in the mice by using a heating pad. After $48 \mathrm{~h}$, mice were deeply anesthetized with tribromoethanol and perfused intracardially with Ringer's solution. Brains were quickly removed and frozen on dry ice. Coronal cryosections of the brains (20 $\mu \mathrm{m}$ in thickness) were cut every $400 \mu \mathrm{m}$ (20-22 sections per brain) and stained with a silver technique. ${ }^{9}$ The size of the ischemic lesion was determined using Scion Image software (Scion Corporation, Frederick, MD, USA) and corrected for brain edema. To evaluate sensorimotor function we performed corner tests as described previously. ${ }^{24}$ Mice were randomly assigned to treatment groups and the investigator was unaware of the treatment or genotype.

Real-time PCR. Primary cortical neurons on six-well plates were lysed and RNA was isolated using RNeasy Mini Kit (Qiagen, Hilden, Germany) according to the manufacturer's protocol. RNA $(2 \mu \mathrm{g})$ was transcribed with Moloney murine leukemia virus reverse transcriptase and random hexamer primers. For real-time PCR the following primers were used: cyclophilin A forward, $5^{\prime}$-AGGT CCTGGCATCTTGTCCAT-3'; cyclophilin A reverse, $5^{\prime}$-GAACCGTTTGTGTT TGGTCCA-3'; COX-2 forward, 5'-TGAGCAACTATTCCAAACCAGC-3'; COX-2 reverse, $5^{\prime}$-GCACGTAGTCTTCGATCACTATC-3'; p40 phox forward, $5^{\prime}$-GTCAT CGAGGTCAAAACAAAAGG-3'; p40 phox reverse, $5^{\prime}$-GCCCATGTAGACTTTGG CTG-3'; NOX-2 forward, 5'-GGTTTATGATGATGGGCCTAA-3'; NOX-2 reverse, 5'-GCACTGGAACCCCTGAGAAA-3'; ASK-1 forward, 5'-ACTCCAGTCCCTGAA GGAAAT-3', ASK-1 reverse, 5'-CAGTAGACCTTGTTGTGTGGTG-3'; MEKK3 forward, 5'-ATAAGGACACAGGTCACCCAA-3'; MEKK3 reverse, $5^{\prime}$-TGCT CCACATCTTCGTATCTCA-3'. Real-time PCR was performed according to the following protocol: $10 \mathrm{~min}$ at $95^{\circ} \mathrm{C}, 15 \mathrm{~s}$ at $95^{\circ} \mathrm{C}$, and $1 \mathrm{~min}$ at $60^{\circ} \mathrm{C}$ (40 cycles). Amplification was quantified using Absolute Blue qPCR SYBR Green Mix (Thermo Scientific, Waltham, MA, USA). Quantified results for individual cDNAs were normalized to cyclophilin $A$ using the $\Delta \Delta C$ t method. The purity of the amplified products was checked by the dissociation curve.

TAK1 kinase assay. Cell lysates were prepared using $100 \mu$ lysis buffer (50 mM Tris-HCl, pH 7.4, $150 \mathrm{mM} \mathrm{NaCl}, 1 \%$ NP-40, $0.25 \%$ sodium deoxycholate, $1 \mathrm{mM}$ EDTA, $2 \mu \mathrm{M}$ leupeptin, and $1 \mu \mathrm{M}$ PMSF) per well. Soluble parts were separated and diluted with PBS to $1 \mathrm{ml}$. Then, lysates were cleared by incubation with $100 \mu$ of protein-G-sepharose beads at $4^{\circ} \mathrm{C}$ for $15 \mathrm{~min}$. Protein concentration was determined by Bradford assay, using Roti-Quant (Roth). Next, $500 \mu \mathrm{g}$ of protein lysates was incubated with $1 \mu \mathrm{l}$ of anti-TAK1-antibody for $2 \mathrm{~h}$ at $4^{\circ} \mathrm{C}$ under gentle rocking. Immunocomplexes were captured with $100 \mu$ protein-G-sepharose and $2 \mu \mathrm{g}$ of anti-rabbit-lgG at $4^{\circ} \mathrm{C}$. Immunocomplexes were washed three times in PBS and $20 \mu$ lof this suspension was used for kinase reaction.

The cDNA coding for human MKK6 was cloned into the pGEX-4T vector (Amersham Pharmacia Biotech, Freiburg, Germany) to produce GSH S-transferase (GST) fusion proteins. Recombinant protein was purified using Glutathione Sepharose 4B (Amersham). For the kinase reaction, $20 \mu \mathrm{l}$ of the isolated immunocomplexes was mixed with $1 \mu \mathrm{g}$ purified MKK6-GST protein, $1 \mu \mathrm{Ci} \gamma^{32} \mathrm{P}$ ATP, $20 \mathrm{mM}$ Tris- $\mathrm{HCl}, \mathrm{pH} 7.5,2 \mathrm{mM}$ EGTA, and $10 \mathrm{mM} \mathrm{MgCl}$, and incubated at $30^{\circ} \mathrm{C}$ for $10 \mathrm{~min}$. Reaction was stopped by heating to $95^{\circ} \mathrm{C}$ for $5 \mathrm{~min}$. Finally, samples were separated by SDS-PAGE analysis. Because of the partial degradation, MKK6 formed a double band in some experiments.

Immunoblotting. Neurons were pretreated for either 10 days or at $1 \mathrm{~h}$ with the TAK1 inhibitor OZ (600 nM) or DMSO. Then, $10^{6}$ neurons were lysed at different time points after OGD. Neuronal lysates were resolved by $6-12 \%$ SDS-polyacrylamide gel electrophoresis. TAK1 was detected using rabbit anti-
TAK1 or the exon 2-specific antibody. Phospho-c-Jun, c-Jun, phospho-p38/MAPK, and p38/MAPK were detected with rabbit anti-phospho-c-Jun and anti-c-Jun, anti-phospho-p38/MAPK (Thr 180/Tyr 182), and anti-p38/MAPK antibodies (Cell Signaling, Danvers, MA, USA). I $\mid \kappa \alpha$ was detected using goat anti- $\mid \kappa \mathrm{B} \alpha$ (Santa Cruz). After detection of phosphorylated proteins membranes were stripped $(100 \mathrm{mM} \mathrm{NaOH}, 10 \mathrm{~min}$ ) and reprobed with antibodies against non-phosphorylated proteins. For immunoblotting, the ischemic region of the cortex was dissected and lysed in RIPA buffer ( $150 \mathrm{mM} \mathrm{NaCl}, 50 \mathrm{mM}$ Tris- $\mathrm{HCl}$ (pH 7.4), $10 \mathrm{mM}$ EDTA, $0.1 \%$ SDS, $1 \%$ triton X-100, $0.5 \%$ sodium deoxycholate, $0.5 \mathrm{mM}$ PMSF) supplemented with phosphatase inhibitor cocktails $1+2$ (Sigma, Steinheim, Germany) and protease inhibitor tablets (Roche Applied Science).

Quantification of superoxide anions by DHE and GSH-Glo assay. Immediately after OGD, DHE (Molecular Probes, Darmstadt, Germany; $5 \mu \mathrm{M}$ ) was added to neuronal cultures in neurobasal medium without phenol red and incubated for $30 \mathrm{~min}$ at $37^{\circ} \mathrm{C}$. Then, cells were washed, fixed, and stained for DAPI. For evaluation, the percentage of nuclei stained red was quantified using ImageJ software (Scion, Frederick, MD, USA).

The amount of GSH in the cytoplasm of primary cortical neurons was analyzed using GSH-Glo Glutathione Assay (Promega, Mannheim, Germany) according to the manufacturer's instructions. Briefly, primary cortical neurons were lysed $30 \mathrm{~min}$ after OGD in $100 \mu$ lysis and reaction buffer per well. Then, $10 \mu$ lof lysate was mixed with $100 \mu \mathrm{l} \mathrm{GSH}$-Glo reagent in a white-walled 96-well plate. The mixture was incubated for $30 \mathrm{~min}$, and then $100 \mu \mathrm{l}$ of luciferin detection reagent was added to each well, mixed, and incubated for $15 \mathrm{~min}$. Samples were measured with a luminometer.

Statistical analyses. Data are expressed as mean \pm S.E.M. Student's $t$-test was used to compare the two groups, and one-way ANOVA followed by NewmanKeuls post hoc test to compare more than two groups.

\section{Conflict of Interest}

The authors declare no conflict of interest.

Acknowledgements. We thank Nadine Gehrig and Hans-Peter Gensheimer for expert technical assistance, Dr Takehana, Kawasaki, Japan, for providing OZ, and Dr Nettelbeck, Heidelberg, Germany, for providing Ad-GFP. This study was supported by a grant of the Deutsche Forschungsgemeinschaft to MS (SCHW 416/5-1) and by funding from the European Union's Seventh Framework Program FP7 (2007-2013) under grant agreements 201024 and 202213 (European Stroke Network).

1. Donnan GA, Fisher M, Macleod M, Davis SM. Stroke. Lancet 2008; 371: 1612-1623.

2. Endres M, Engelhardt B, Koistinaho J, Lindvall O, Meiairs S, Mohr JP et al. Improving outcome after stroke: overcoming the translational roadblock. Cerebrovasc Dis 2008; 25 : 268-278.

3. Ridder DA, Schwaninger M. NF-kappaB signaling in cerebral ischemia. Neuroscience 2009; 158: 995-1006.

4. Ghosh A, Roy A, Liu X, Kordower JH, Mufson EJ, Hartley DM et al. Selective inhibition of NF-\{kappa\}B activation prevents dopaminergic neuronal loss in a mouse model of Parkinson's disease. Proc Natl Acad Sci USA 2007; 104: 18754-18759.

5. Zhang F, Qian L, Flood PM, Shi JS, Hong JS, Gao HM. Inhibition of IkappaB kinase-beta protects dopamine neurons against lipopolysaccharide-induced neurotoxicity. J Pharmacol Exp Ther 2010; 333: 822-833.

6. Herrmann O, Baumann B, De Lorenzi R, Muhammad S, Zhang W, Kleesiek J et al. IKK mediates ischemia-induced neuronal cell death. Nat Med 2005; 11: 1322-1329.

7. Khoshnan A, Ko J, Watkin EE, Paige LA, Reinhart PH, Patterson PH. Activation of the IkappaB kinase complex and nuclear factor-kappaB contributes to mutant huntingtin neurotoxicity. J Neurosci 2004; 24: 7999-8008.

8. Schneider A, Martin-Villalba A, Weih F, Vogel J, Wirth T, Schwaninger M. NF- $\kappa B$ is activated and promotes cell death in focal cerebral ischemia. Nat Med 1999; 5: 554-559.

9. Inta I, Paxian S, Maegele I, Zhang W, Pizzi M, Spano P et al. Bim and Noxa are candidates to mediate the deleterious effect of the NF-\{kappa\}B subunit RelA in cerebral ischemia. J Neurosci 2006; 26: 12896-12903.

10. Irving $E A$, Bamford M. Role of mitogen- and stress-activated kinases in ischemic injury. $J$ Cereb Blood Flow Metab 2002; 22: 631-647.

11. Borsello T, Clarke PG, Hirt L, Vercelli A, Repici M, Schorderet DF et al. A peptide inhibitor of c-Jun $\mathrm{N}$-terminal kinase protects against excitotoxicity and cerebral ischemia. Nat Med 2003; 9: 1180-1186.

12. Waetzig V, Herdegen T. Context-specific inhibition of JNKs: overcoming the dilemma of protection and damage. Trends Pharmacol Sci 2005; 26: 455-461. 
13. Barone FC, Irving EA, Ray AM, Lee JC, Kassis S, Kumar S et al. Inhibition of p38 mitogenactivated protein kinase provides neuroprotection in cerebral focal ischemia. Med Res Rev 2001; 21: 129-145.

14. Won M, Park KA, Byun HS, Sohn KC, Kim YR, Jeon J et al. Novel anti-apoptotic mechanism of A20 through targeting ASK1 to suppress TNF-induced JNK activation. Cell Death Differ 2010; 17: 1830-1841.

15. Papa S, Bubici C, Zazzeroni F, Pham CG, Kuntzen C, Knabb JR et al. The NF-kB-mediated control of the JNK cascade in the antagonism of programmed cell death in health and disease. Cell Death Differ 2006; 13: 712-729.

16. Nijboer $\mathrm{CH}$, Heijnen $\mathrm{CJ}$, Groenendaal F, van Bel F, Kavelaars A. Alternate pathways preserve tumor necrosis factor-\{alpha\} production after nuclear factor-\{kappa\}B inhibition in neonatal cerebral hypoxia-ischemia. Stroke 2009; 40: 3362-3368.

17. Shim J-H, Xiao C, Paschal AE, Bailey ST, Rao P, Hayden MS et al. TAK1, but not TAB1 or TAB2, plays an essential role in multiple signaling pathways in vivo. Genes Dev 2005; 19 : 2668-2681.

18. Sato S, Sanjo H, Takeda K, Ninomiya-Tsuji J, Yamamoto M, Kawai T et al. Essential function for the kinase TAK1 in innate and adaptive immune responses. Nat Immunol 2005; 6: 1087-1095.

19. Tang $M$, Wei $X$, Guo $Y$, Breslin $P$, Zhang $S$, Zhang $S$ et al. TAK1 is required for the survival of hematopoietic cells and hepatocytes in mice. J Exp Med 2008; 205: 1611-1619.

20. Omori E, Matsumoto K, Sanjo H, Sato S, Akira S, Smart RC et al. TAK1 is a master regulator of epidermal homeostasis involving skin inflammation and apoptosis. J Biol Chem 2006; 281: 19610-19617.

21. Bettermann K, Vucur M, Haybaeck J, Koppe C, Janssen J, Heymann F et al. TAK1 suppresses a NEMO-dependent but NF-[kappa]B-independent pathway to liver cancer. Cancer Cell 2010; 17: 481-496.

22. Yamaguchi K, Shirakabe K, Shibuya H, Irie K, Oishi I, Ueno N et al. Identification of a member of the MAPKKK family as a potential mediator of TGF-beta signal transduction. Science 1995; 270: 2008-2011.

23. Ninomiya-Tsuji J, Kajino T, Ono K, Ohtomo T, Matsumoto M, Shiina M et al. A resorcylic acid lactone, 5Z-7-oxozeaenol, prevents inflammation by inhibiting the catalytic activity of TAK1 MAPK kinase kinase. J Biol Chem 2003; 278: 18485-18490.

24. Lubjuhn J, Gastens A, von Wilpert G, Bargiotas P, Herrmann O, Murikinati S et al. Functional testing in a mouse stroke model induced by occlusion of the distal middle cerebral artery. J Neurosci Methods 2009; 184: 95-103.

25. Erdmann G, Berger S, Schutz G. Genetic dissection of glucocorticoid receptor function in the mouse brain. J Neuroendocrinol 2008; 20: 655-659.

26. Mehta SH, Dhandapani KM, De Sevilla LM, Webb RC, Mahesh VB, Brann DW. Tamoxifen, a selective estrogen receptor modulator, reduces ischemic damage caused by middle cerebral artery occlusion in the ovariectomized female rat. Neuroendocrinology 2003; 77 : 44-50.

27. von Knethen A, Callsen D, Brune B. Superoxide attenuates macrophage apoptosis by $\mathrm{NF}-\{$ kappa\}B and AP-1 activation that promotes cyclooxygenase-2 expression. $\mathrm{J}$ Immunol 1999; 163: 2858-2866.

28. Anrather J, Racchumi G, ladecola $\mathrm{C}$. NF- $\kappa$ B regulates phagocytic NADPH oxidase by inducing the expression of gp91phox. J Biol Chem 2006; 281: 5657-5667.

29. Manea A, Manea SA, Gafencu AV, Raicu M, Simionescu M. AP-1-dependent transcriptional regulation of NADPH oxidase in human aortic smooth muscle cells: role of p22phox subunit. Arterioscler Thromb Vasc Biol 2008; 28: 878-885.

30. Franco R, Cidlowski JA. Apoptosis and glutathione: beyond an antioxidant. Cell Death Differ 2009; 16: 1303-1314.

31. Liu Q, Wilkins BJ, Lee YJ, Ichijo H, Molkentin JD. Direct interaction and reciprocal regulation between ASK1 and calcineurin-NFAT control cardiomyocyte death and growth. Mol Cell Biol 2006; 26: 3785-3797.

32. Stetler RA, Cao G, Gao Y, Zhang F, Wang S, Weng Z et al. Hsp27 protects against ischemic brain injury via attenuation of a novel stress-response cascade upstream of mitochondrial cell death signaling. J Neurosci 2008; 28: 13038-13055.

33. Takeda K, Noguchi T, Naguro I, Ichijo H. Apoptosis signal-regulating kinase 1 in stress and immune response. Ann Rev Pharmacol Toxicol 2008; 48: 199-225.

34. Candelario-Jalil E, Alvarez D, Merino N, León OS. Delayed treatment with nimesulide reduces measures of oxidative stress following global ischemic brain injury in gerbils. Neurosci Res 2003; 47: 245-253.

35. Abramov AY, Scorziello A, Duchen MR. Three distinct mechanisms generate oxygen free radicals in neurons and contribute to cell death during anoxia and reoxygenation. J Neurosci 2007; 27: 1129-1138.

36. Kunz A, Anrather J, Zhou P, Orio M, ladecola C. Cyclooxygenase-2 does not contribute to postischemic production of reactive oxygen species. J Cereb Blood Flow Metab 2006; 27: 545-551.

37. Nogawa S, Zhang F, Ross ME, ladecola C. Cyclo-oxygenase-2 gene expression in neurons contributes to ischemic brain damage. J Neurosci 1997; 17: 2746-2755.

38. Chen H, Song YS, Chan PH. Inhibition of NADPH oxidase is neuroprotective after ischemia-reperfusion. J Cereb Blood Flow Metab 2009; 29: 1262-1272.

39. Araki K, Kawauchi K, Tanaka N. IKK/NF-[kappa]B signaling pathway inhibits cell-cycle progression by a novel $\mathrm{Rb}$-independent suppression system for $\mathrm{E} 2 \mathrm{~F}$ transcription factors. Oncogene 2008; 27: 5696-5705.

40. Omori E, Morioka S, Matsumoto K, Ninomiya-Tsuji J. TAK1 regulates reactive oxygen species and cell death in keratinocytes, which is essential for skin integrity. J Biol Chem 2008; 283: 26161-26168. 